\title{
Children with Upper Airway Dysfunction: At Risk of Obstructive Sleep Apnea
}

\author{
Carlos Sisniega ${ }^{1} \quad$ Umakanth Katwa ${ }^{2}$
}

Address for correspondence Umakanth Katwa, MD, Division of Respiratory Diseases, Boston Children's Hospital, Harvard Medical School, 300 Longwood Ave., Boston, MA, 02115, United States (e-mail: Umakanth.katwa@childrens.harvard.edu).

J Child Sci 2019;9:e59-e67.

\begin{abstract}
Keywords

- upper airway

- sleep apnea

- sleep breathing

- tongue base

- snoring

- pathophysiology

Obstructive sleep apnea is characterized by prolonged partial upper airway obstruction or intermittent complete obstruction that disrupts normal ventilation during sleep and alters normal sleep patterns. Patients with obstructive sleep apnea tend to develop neurocognitive, cardiovascular, behavioral, attention issues, and poor academic performance. Therefore, it is essential to diagnose and treat obstructive sleep apnea early and avoid significant and long-lasting adverse outcomes. Most commonly, upper airway obstruction is caused by enlarged lymphoid tissues within the upper airway, and therefore adenotonsillectomy is considered as the first-line treatment of obstructive sleep apnea in children. Fifty to $70 \%$ of patients who have obstructive sleep apnea and treated by surgery are not entirely cured on follow-up polysomnography. In light of this, it is recommended that patients with suspected obstructive sleep apnea undergo a thorough evaluation, and all potential risk factors are identified and treated. The purpose of this review is to familiarize pediatricians with developmental, anatomical, and physiological risk factors involved in the development of obstructive sleep apnea. Additionally, we will present an array of evaluation techniques that can offer adequate assessment of the patient's upper airway anatomy and physiology.
\end{abstract}

\section{Introduction}

Obstructive sleep apnea (OSA) is a subtype of a larger class of sleep-related breathing disorders and is characterized by prolonged partial upper airway obstruction or intermittent complete obstruction that disrupts normal ventilation during sleep and alters normal sleep patterns. ${ }^{1}$ The prevalence of OSA is 2 to $4 \%$ among the general pediatric population. However, this prevalence changes with age and peaks between 1.5 and 5 years of age, coinciding with the peak growth of adenotonsillar tissues in the upper airway. In patients with Down's syndrome (DS), the prevalence can be as high as $66 \%{ }^{2}$ OSA is characterized by nighttime

received

April 2, 2019

accepted

April 3, 2019
Issue Theme Sleep Disorders in Children; Guest Editors: David Gozal, MD, MBA, PhD (Hon), and Leila Kheirandish-Gozal, MD, MSc. symptoms such as frequent snoring (typically $\geq 3$ nights per week), labored breathing during sleep, mouth breathing, witnessed apneas, choking- or gasping-induced arousals, sleep enuresis, and restless sleep. Morning symptoms may include headaches on awakening, excessive daytime sleepiness, attention deficit/hyperactive disorder, and behavioral and learning problems. Also, these patients tend to have neurocognitive, cardiovascular, behavioral, attention issues, and poor academic performance. ${ }^{3-6}$ Risk factors encountered in children with OSA include adenoid and tonsil hypertrophy, turbinate hypertrophy, obesity, and other craniofacial abnormalities such as micrognathia/retrognathia and higharched palate. ${ }^{7}$ Therefore, it is essential to diagnose and treat
Copyright @ 2019 Georg Thieme Verlag License terms KG Stuttgart · New York 
OSA syndrome early and to avoid significant and long-lasting adverse outcomes.

OSA is caused by a complex interaction of structural, functional, and behavioral elements influencing the airways. The most common anatomical cause of upper airway obstruction is enlarged lymphoid tissues within the upper airway, and therefore adenotonsillectomy (AT) is considered as the first-line treatment of OSA in children. ${ }^{7}$ Yet, it has been shown in several studies and subsequently by a meta-analysis that AT is not very effective in curing OSA (i.e., normalizing the sleep study and all respiratory disturbances during sleep) in most children. For example, in a prospective study involving 56 children (age: 16 months to 12.5 years), Guilleminault et al showed that in patients who had undergone AT or orthodontic intervention, 29 (51.7\%) children still had an apnea-hypopnea index (AHI) of $>1$ event per hour of sleep and 12 (41.3\%) patients had an AHI of $>5$ events per hour of sleep. ${ }^{8}$ Additionally, these researchers found that when the recommended multidisciplinary approach was not followed, rates of failures (i.e., AHI $>1$ event per hour of sleep) were much higher ( 88.9 vs. $10 \%){ }^{8}$ Two years later, Tauman et al showed in a prospective series of 110 children that obesity and OSA severity were important factors in the resolution of OSA after AT but also confirmed that the "cure rate" after AT was exceedingly low and averaged approximately $30 \%{ }^{9}$ Later in 2010, Bhattacharjee et al in a multicenter retrospective study looked at 578 children who underwent AT. They found that only 157 (27.2\%) had complete resolution of their OSA by PSG (i.e., AHI < one event per hour of sleep) after AT. Additionally, they found that age $>7$ years, obesity, and a history of asthma contributed to the persistence of OSA after $\mathrm{AT}^{10}$ These results reflect highly complex interactions of various factors involved in the pathogenesis of OSA. Hence, a comprehensive multidisciplinary evaluation for the management of OSA is critical for an accurate and personalized evaluation. In this article, we will review the various factors involved in the development of upper airway dysfunction and discuss some diagnostic strategies to evaluate these children.

\section{Airway Development}

To better understand the development of airway dysfunction, it is critical to acquire a good understanding of upper airway development. There are critical periods during airway development that can lead to dysfunction, which, if unrecognized or untreated, can lead to significant dysmorphism, necessitating surgical intervention. The upper airway extends from the nasal opening and mouth to the vocal cords in the larynx. The upper airway is a complex structure composed of various muscles from different embryological origins and is involved in several functions such as breathing, swallowing, and speech. Craniofacial development begins during the fourth week of embryonic life with the migration of neural crest cells, which progressively develop into many facial bones known as the maxillary and mandibular prominence through the activations of several genes such as HOX, FGF, and Pax-6. ${ }^{11}$ Following anatomical development, functional development of the swallowing and sucking reflex begins. These suck/swallow reflexes along with postnatal nasal breathing are crucial for the optimal development of craniofacial and upper airway anatomy and function.

\section{Prematurity}

Studies have shown that premature infants are at an increased risk of upper airway dysfunction due to decreased upper airway muscle tone and high nasal airway resistance. In addition, premature infants tend to have smaller airways. In one study, $8.1 \%$ of premature patients were reported to manifest snoring for three or more nights per week, ${ }^{12}$ and in another study, children with a history of prematurity were more likely to snore and undergo AT. Additionally, patients with a history of prematurity had a significantly higher AHI (AHI $>5$ [0.9\% in full-term vs. $4.3 \%$ in premature infants]) and significantly lower oxygen saturation during sleep. ${ }^{13}$ Preliminary evidence also shows that premature infants with high and narrow palates, with low muscle tone, and who are mouth-breathers create an inflammatory environment, leading to an increased risk of allergies and enlarged adenoids and tonsils. ${ }^{14}$ Infants are obligatory nasal breathers, and therefore premature infants who are at a high risk of increased nasal resistance due to underdeveloped nasomaxillary complex are also at a much higher risk of OSA. In addition, low muscle tone seen in these infants predisposes to habitual mouth breathing, leading to further craniomaxillary complex maldevelopment. Although anatomical problems tend to play a major role in causing OSA in premature infants, some studies suggest that blunted ventilatory responses to hypoxia during sleep in premature infants may also worsen gas exchange which can cause central respiratory pauses following desaturation. ${ }^{15}$

\section{Anatomical Consideration of Upper Airway Dysfunction}

\section{Nose}

Previously stated embryological structures are necessary for the development of the base of the nasal cavity and the base and roof of the oral cavity. After birth, facial structures continue to develop. By age six, the face is $60 \%$ the size of the adult face dimensions, reaching maximal growth by the age of 12 years. During this time, facial growth is governed by the growth of the cranial base through endochondral ossification, which is under the influence of many physiological conditions. ${ }^{11}$ Breathing through the nasal cavity, the primary route of breathing in infants, provides stimulus for the proper growth of the sinuses, nasal, and oral cavity. ${ }^{16}$ In nasal airflow, two areas of major resistance are encountered: the nasal valve and the turbinate mucosa. These factors help in the proper development of the palate and dental arches. Cyclic changes in the blood supply to the mucosa alter the space for airflow through each nasal chamber; a phenomenon referred to as the nasal cycle. ${ }^{16}$ The nasal cycle can be altered in children with nasal allergies or nasal blockage. More detailed description about nasal airflow can be found elsewhere. ${ }^{17}$

Obstructions at the level of the nasal cavity can cause a significant degree of obstructive sleep-disordered breathing 
resulting in sleep fragmentation. In adult studies, nasal obstruction has been shown to reduce time in deep nonrapid eye movement (NREM; stage N3) sleep and increase the incidence of apneic episodes. ${ }^{18}$ Based on the Starling law, upstream obstruction at the nasal level increases the turbulence of airflow and leads to increased propensity for airway collapse at the pharyngeal level. In children with allergic rhinitis, studies have shown increased incidence of obstructive SDB, which can be reduced by the administration of intranasal steroids and by other measures that can help increase nasal patency and reduce upper airway resistance. ${ }^{19,20}$ During childhood, altering normal nasal breathing aerodynamics can lead to craniofacial skeletal abnormalities. In these children, the floor of the nasal cavity (palate) moves up (high arch palate), and the maxilla is more constricted and retruded. Additionally, there is lowering of the inferior portion of the posterior nasal fossa, causing impingement on the pharyngeal region, thus increasing the chances of velopharyngeal obstruction. ${ }^{21}$ As a consequence of these changes, children can be easily distinguished in the clinic by the presence of "adenoid facies," which include maxillary growth restriction, incomplete lip seal, narrow upper dental arch (posterior crossbite), crowded teeth, increased lower anterior face height, open mouth posture, a steep mandibular plane angle, and a retrognathic mandible. ${ }^{21}$

\section{Oropharynx}

Mastication and swallowing are primary functions of the oral cavity, and the oral cavity is not specifically programmed for normal breathing. As noted earlier, oral breathing is a default route to breathe in children with nasal obstruction. The oropharynx begins to develop in the second month of pregnancy. During this time, tongue placement on the roof of palate allows for closure of the mouth. This developmental process is a critical part in upper airway development as it sets up a suction apparatus able to create negative pressure and thus facilitate a physiological environment for breathing, sucking, swallowing, and phonation. ${ }^{11}$ The location of the tongue on the roof of the mouth in resting position acts like a natural expander to the hard palate and helps in normal maxillary and dental arch development. Integrated into the development of the oropharynx is positioning of the hard and soft palate relative to the tongue and adenoid tissues, which are positioned posteriorly to the nasopharynx. As discussed earlier, children with nasal obstruction become mouth-breathers and are predisposed to low tongue posture, which may contribute to a narrow palate and a reduction of the space for the tongue in the oropharynx (small mouth size or relative macroglossia). Hence, during the development of the oropharynx, if physiological homeostasis in breathing is altered, obstruction to breathing can happen at various locations such as tonsils, soft palate, and tongue base, and then at the laryngeal level.

To summarize the site and type of obstruction, Kezirian et al developed the VOTE (velum, oropharynx lateral walls, tongue base, epiglottis) classification. ${ }^{22}$ Based on observations from drug-induced sleep endoscopies (DISEs), the VOTE classification focuses on the most common sites of obstruction and their configuration. The structures included in the classification are the velum, oropharynx and lateral walls, tongue base, and epiglottis. The degree of obstruction can be classified into no obstruction, partial obstruction, or complete obstruction. Finally, the configuration of airway collapse can be stated as anteroposterior (AP), lateral, or concentric type (- Table 1). ${ }^{22}$

\section{Velum}

Velum or soft palate collapse has been shown to occur in patients with OSA. Soft palate and uvula typically vibrate during snoring. In children with long-standing snoring, these structures tend to be enlarged, swollen, inflamed, and redundant. Therefore, in this situation, the soft palate can collapse on the velopharynx on inspiration and lead to obstructive events. The velum and the uvula can also prolapse into the velopharynx during expiration, causing air to escape through the mouth. ${ }^{23}$ This palatal prolapse during DISE has been shown to limit expiratory flow. Researchers concluded that the phenomenon of expiratory palatal prolapse could be caused by an elongated palate, a swollen uvula, or excess soft palatal tissue. ${ }^{23}$ This increase in expiratory pressure and fluttering of tissue could lead to oral exhalation. Additionally, it was also hypothesized that this mechanism could lead patients to become mouthbreathers, as it has been shown that children who mouthbreathe are at a higher risk of tongue base collapse. ${ }^{23}$ Finally, during inspiration, there is a decrease in the driving pressure to inspiratory flow due to the dislodgement of the velum, leading to shallow breaths that may be categorized as hypopneas. ${ }^{23}$

Although video-assisted endoscopy can readily identify the site of upper airway collapse, researchers have also shown that less expensive and less invasive techniques can be used to point at the place of obstruction. Negative effort dependence has been shown to be an excellent tool to understand which part of the upper airway structure is involved in the collapse. ${ }^{24}$ Additionally, this tool could help in the evaluation of patients who do not tolerate continuous

Table 1 The VOTE classification (reproduced with permission from authors)

\begin{tabular}{|l|l|l|l|l|}
\hline Structure & \multirow{2}{*}{$\begin{array}{l}\text { Degree of } \\
\text { obstruction }^{\mathrm{a}}\end{array}$} & \multicolumn{3}{|c|}{ Configuration $^{\mathrm{b}}$} \\
\cline { 3 - 5 } & & AP & Lateral & Concentric \\
\hline Velum & & & & \\
\hline $\begin{array}{l}\text { Oropharynx } \\
\text { lateral walls }\end{array}$ & & & & \\
\hline Tongue base & & & & \\
\hline Epiglottis & & & & \\
\hline
\end{tabular}

Abbreviation: AP, anteroposterior.

Note: for each structure, there should be a classification of the degree of obstruction and configuration of obstruction. Open boxes reflect the potential configuration that can be visualized related to a specific structure. Black boxes reflect the fact that a specific structure configuration cannot be seen.

${ }^{a}$ Degree of obstruction has one number for each structure: 0 , no obstruction (no vibration); 1, partial obstruction (vibration); 2, complete obstruction (collapse); X, not visualized.

${ }^{\mathrm{b}}$ Configuration noted for structures with degree of obstruction greater than 0 .

'Oropharynx obstruction can be distinguished as related solely to the tonsils or including the lateral walls, with or without tonsil component. 
positive airway pressure (CPAP), and accordingly, other therapies could be offered to these patients.

\section{Tongue}

The tongue plays an important role in the development of upper airway obstruction. As part of the inspiratory phasic upper airway muscle dilator (along with influencing the hyoid position and the muscles of the palate), the genioglossal muscle is tensed during inspiration, increasing negative pressure (i.e., decreasing $P_{\text {crit }}$ ). As previously stated, as the patient goes into NREM sleep, glossal and pharyngeal muscle tonic activity is reduced, causing an increase in the capacitance and collapsibility of the upper airway. ${ }^{25,26}$ This mechanism for collapsibility has been shown to be augmented in patients with OSA. Additionally, the reflex that blunts this response in normal patients also affects the patients with OSA. ${ }^{27}$ For example, the genioglossus muscle has been shown to function at $40 \%$ of its capacity in patients with OSA. ${ }^{28}$ Therefore, tongue base obstruction is a major site of obstruction in children with OSA. Previous research has shown that upper airway muscle tone, mandible size, and tongue volume are all variables that influence the development of obstruction. ${ }^{29}$ As seen in many of the studies of OSA in patients with DS, the decreased ratio of mandibular area to tongue area can cause airway overcrowding and posterior displacement of tongue, consequently increasing the risk of obstruction at the level of the tongue base. ${ }^{30}$ In one study, the researchers found that the previously stated ratio by magnetic resonance imaging (MRI) caused airway obstruction by glossoptosis and hypopharyngeal collapse in nearly twothirds of children with OSA and DS after AT. ${ }^{31}$ Soft tissue surrounding the upper airway also plays an essential role in upper airway collapse. It has been clearly shown that fatty deposition in the tongue and around the airway is a risk factor for OSA. ${ }^{32,33}$

\section{Epiglottis}

At the level of the epiglottis, laxity has also been shown to obstruct the upper airway. The epiglottis might fold posteriorly or laterally and cause airway obstruction. ${ }^{23}$ In adults, this can happen in $12 \%$ or more of patients with OSA. Sleepstate laryngomalacia is a distinct condition where the epiglottis tends to become floppy and completely collapses, blocking airflow. However, in awake nasal endoscopy, the larynx appears normal. This is readily seen in pediatric patients diagnosed with laryngomalacia. ${ }^{34}$ As the upper airway becomes obstructed, changes in the oropharynx occur, which are often missed by the clinicians. Specifically, with prolonged obstructive breathing, children develop cephalometric parameter changes that have been associated with OSA.

\section{Obstructive Breathing and Changes in Skeletal and Dental Structures}

Luzzi et al measured several parameters in patients with primary snoring using lateral cephalograms. ${ }^{35}$ In this study, researchers measured sagittal measurements such as the sella-nasion-A (SNA) point angle, the sella-nasion-B (SNB) point angle, and the $A$ point-nasion- $B$ point angle (ANB) ( - Fig. 1A). These measurements defined the AP relationship between the maxilla and the mandible. In the vertical axis, the Frankfurt mandibular angle (FMA) was measured. The FMA angle is the angle between the Frankfurt horizontal plane and the mandibular plane, a well-established measurement of total facial divergence ( $\mathbf{- F i g}$. 1B). The FMA angle is considered normal between 20 and 30 degrees. These investigators
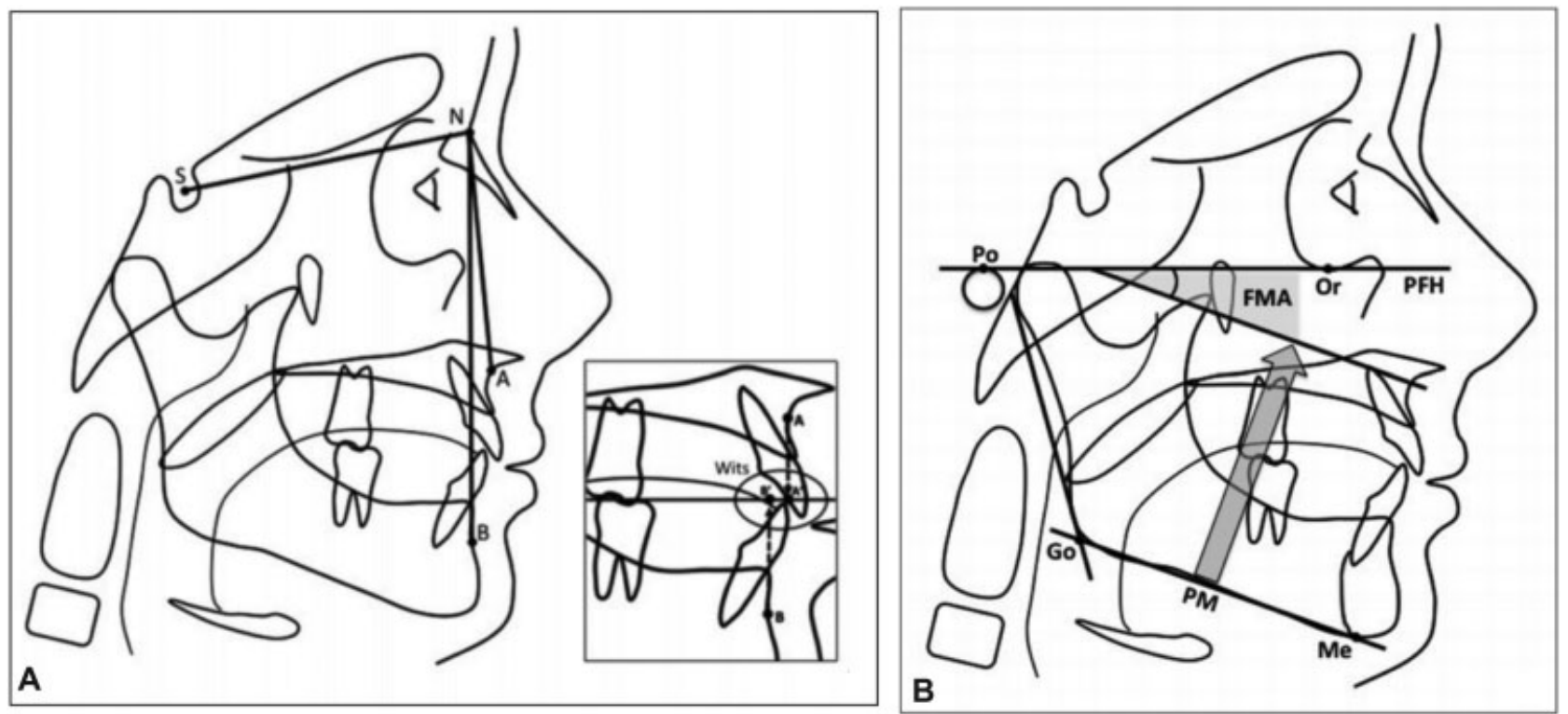

Fig. 1 Cephalometric parameters. (A) Frankfurt mandibular angle (FMA) measures the angle between the Frankfurt horizontal plane and the mandibular plane. (B) SNA, "sella-nasion-A point" angle, measures the anteroposterior relationship of maxillary basal arch on anterior cranial base. SNB, "sella-nasion-B point" angle, measures the anterior limit of the mandibular basal arch in relation to the anterior cranial base. Go, gonion or mandibular angle; Me, mental protuberance; PM, plane of mandible; PFH: plane of Frankfurt. Reproduced with permission from Luzzi et al. ${ }^{35}$ 
showed that the FMA angle was the only orthodontic parameter that was statistically associated with the level of airway obstruction. When the FMA angle is increased, the degree of airway obstruction is also increased. ${ }^{35}$ Likewise, other studies have shown that patients who are mouth-breathers develop specific phenotypic characteristics that should alert physicians to evaluate these children for upper airway dysfunction. Pacheco et al studied the prevalence of morphological changes in 520 healthy children, ranging in age from 7 to 12 years old. Within their population, 167 (24.3\%) patients were classified as mouth-breathers. Within this group, $26.1 \%$ had excessive overjet and $17.7 \%$ had an anterior open bite. ${ }^{36}$ Additionally, within the mouth-breather group, 53.9\% had atretic palates. Finally, this group also had a significantly higher prevalence of dolichofacial pattern as well as a convex facial profile. ${ }^{36}$ In adults, Lee et al studied craniofacial morphology with objective facial measurements. As seen in other studies, patients with OSA tended to have shorter and retruded mandible, a smaller area within the mandible, and more fat deposition on the anterior neck. These changes cause patients with OSA to have a brachycephalic head and euryprosopic facial characteristics (more extensive laterally, shorter AP dimensions, and short vertically) when corrected for BMI. ${ }^{37}$

Dental morphology has also been shown to be drastically altered in patients with upper airway obstruction and therefore should be inspected. Pirilä-Parkkinen et al analyzed dental arch morphology in children with SDB and evaluated children who snored, those diagnosed with OSA, and healthy controls. The mean age of the children in the study was 7.2 years (range: 4.3-11.4). Results revealed again that children with OSA had a narrower upper dental arch, increased overjet, reduced overbite, and shorter length of the lower dental arch when compared with nonobstructed controls. ${ }^{38}$ Additionally, this study showed that patients who snore, but are do not fulfill criteria for OSA, still have alteration in their dental arch morphology. Interestingly, many of the patients with OSA did not have enlarged tonsils. ${ }^{38}$

\section{Functional Characteristics of Upper Airway Dysfunction}

\section{Control of Breathing during Sleep}

Breathing is controlled by both peripheral and central inputs. Peripheral chemoreceptors in the carotid and aortic bodies and central chemoreceptors in the ventral area of the medulla oblongata and other areas of the central nervous system sense physiological metabolites (i.e., hydrogen ions and carbon dioxide), which activate the central pattern generator (CPG) area of the brainstem. ${ }^{16}$ Activation of the CPG transmits signals to the respiratory muscles to maintain a normal breathing pattern and gas exchange. During sleep, there is a decrease in the ventilatory drive, shown as a decrease in the slope of the ventilation/CO2 relationship in NREM sleep and rapid eye movement (REM) sleep. Studies have also shown a decrease in hypoxic ventilatory sensitivity during NREM and REM (39\% and 52\%, respectively). ${ }^{39-41}$ Finally, upper airway muscle tone can also be affected during sleep. Because these muscles are under the influence of respiratory chemoreceptors, a decreased or blunted response to hypoxia and hypercapnia allows for increasing resistance of the upper airway during sleep and the emergence of prolonged obstructive events. ${ }^{27}$

\section{Upper Airway Neuromuscular Tone and Critical Closing Pressure ( $\left.\boldsymbol{P}_{\text {crit }}\right)$}

The pharynx constitutes the last portion of the upper airway, made up of mainly skeletal musculature. The patency of the muscular tube is dependent on muscle tone and neurologic activity during sleep. During sleep, this highly compliant muscle tube is under the influence of negative pressures created by the expansion and compression of the diaphragm. Therefore, a small change in pressure can drastically alter its diameter. Critical closing pressure $\left(P_{\text {crit }}\right)$ is the pressure at which the airway collapses and closes completely. Usually, $P_{\text {crit }}$ in the awake state is between $7.4 \mathrm{~cm} \mathrm{H}_{2} \mathrm{O}$, and $-25 \mathrm{~cm} \mathrm{H}_{2} \mathrm{O}$ during sleep. The more negative $P_{\text {crit }}$ during sleep signifies a decrease in compliance of the pharynx and an increase in muscle tonicity during sleep, and hence more stable and less collapsible airways. Conversely, the closer the $P_{\text {crit }}$ gets to zero or becomes positive, the more collapsible the airway becomes. Infants and children tend to have more negative $P_{\text {crit }}$ than adults, and hence their airways are less collapsible compared with adults. ${ }^{42}$ Therefore, in children, OSA is caused mainly by anatomical factors and less by neuromuscular factors. However, patients with OSA tend to have more positive (higher) $P_{\text {crit }}$ during sleep, which predisposes their airway to collapse easily. Huang et al analyzed this effect by measuring several parameters during sleep in patients with OSA and controls. ${ }^{43}$ Their study revealed that patients with OSA had pressure drops during REM sleep, which significantly affected airflow when compared with controls. For example, the mean drop in airflow in the OSA group was $44.33 \pm 14.09 \mathrm{~mL} / \mathrm{seconds}$. Yet, many of these patients were able to compensate and maintain minute ventilation by increasing their respiratory rate. Unfortunately, other studies have revealed that many patients with OSA have decreased compensatory mechanisms, such as the one seen in the previous study. ${ }^{27}$ Therefore, it is essential that patients with OSA maintain lower $P_{\text {crit }}$ pressures to avoid airway collapse during sleep, which is known to cause apneas and hypopneas. Besides the inherent characteristics of the pharynx, many extrinsic characteristics may mitigate or enhance the collapsibility of the pharynx. Adipose tissue deposit in the surrounding connective tissue and around the pharynx in the cervical region, and the neck circumference has been shown to be a risk factor for OSA. ${ }^{44}$ Finally, flexion of the neck has shown to worsen upper airway collapsibility, and neck extension may reduce upper airway collapsibility in patients with OSA. ${ }^{45}$ For this reason, many children with severe OSA will adopt a hyperextended neck posture over a pillow during their sleep.

\section{Loop Gain and Arousal Threshold}

Besides airway pressures, neurologic responses to hypoxia can be measured and have been shown to be related to the 
development of OSA. Loop gain, originally an engineering term, is a method used to measure the stability of a system's feedback loops. In medicine, loop gain has been used to measure the stability of the upper airway during sleep. ${ }^{46}$ This method specifically measures the response of the central nervous system to a disturbance in the upper airway. For example, an excessive ventilatory response (hyperventilation) to hypoxia (disturbance) will decrease $\mathrm{PaCO}_{2}$ due to ventilatory overshooting more than necessary (increased loop gain) and also generate upper airway hypotonia. As a result of this excessive response to hypoxia, upper airway obstruction can be recurring and perpetuating (-Fig. 2). ${ }^{46,47}$ Loop gain has been shown to have a causal relationship with OSA, complementing the theory that OSA has both structural and functional components of the upper airways that need to be considered during the clinical evaluation and treatment. ${ }^{47-49}$ Finally, another pathological disturbance that decreases the nervous system from compensating for hypoxia or airway obstruction is reduced arousal threshold, leading to premature termination of the obstructive events, which impedes the central ventilatory drive from acting on the upper airway, reducing stable breathing during sleep. ${ }^{50}$

\section{Evaluation of Upper Airway Obstruction}

\section{Imaging Studies}

Imaging of upper airways has become an essential part of the evaluation of patients with OSA. As more physicians recognize that patients with OSA might have several sites of obstruction, identifying the anatomy of these patients is essential. Both structural and dynamic evaluation of the airways is essential to assess both size and stability of the airways. Cine magnetic resonance imaging (Cine MRI) has become an integral part of evaluating the upper airway anatomy of patients with DS and OSA. Cine and static MRI allow for proper assessment of the airway and its surrounding tissues. Several studies have shown that MRI is a useful tool for evaluating upper airway anatomy and collapsibility and assessing different sites of obstruction simultaneously in children with DS with OSA. One metaanalysis revealed that patients with OSA had a significantly reduced total volume of the upper airways when compared with controls $\left(1.4 \pm 0.7\right.$ vs. $\left.1.6 \pm 0.7 \mathrm{~cm}^{3}\right)$. A slight reduction in the diameter of airway has a significant impact on the airway resistance. MRI could be used to evaluate the size of lymphoid tissue as well as the cross-sectional area regions surrounding hypertrophied lymphoid tissue. ${ }^{51}$ This meta-analysis revealed that MRI could evaluate differences in upper airway volume in pediatric patients with OSA compared with healthy controls (mean difference: $0.53 \mathrm{~cm}^{3} ; 95 \%$ confidence interval: -1.07 to -0.07 ), as well as soft tissue and skeletal muscle surrounding the airways.

In another study, researchers evaluated the effect of dynamic (cine) MRI to localize and identify the sites of obstruction in the upper airways. ${ }^{52}$ Using either 1.5 - or 3.0-T MRI equipment, sagittal three-dimensional respiratory-triggered fast spin echo, or proton-density sequence, researchers captured sequential videos of patients with OSA. Slices of $1.6 \mathrm{~mm}$ thickness were used to captures images from the tip of the nose anteriorly to the occiput posteriorly, and from the aspect of the nasal airway superiorly to the subglottic trachea inferiorly (-Fig. 3). The researchers used dexmedetomidine

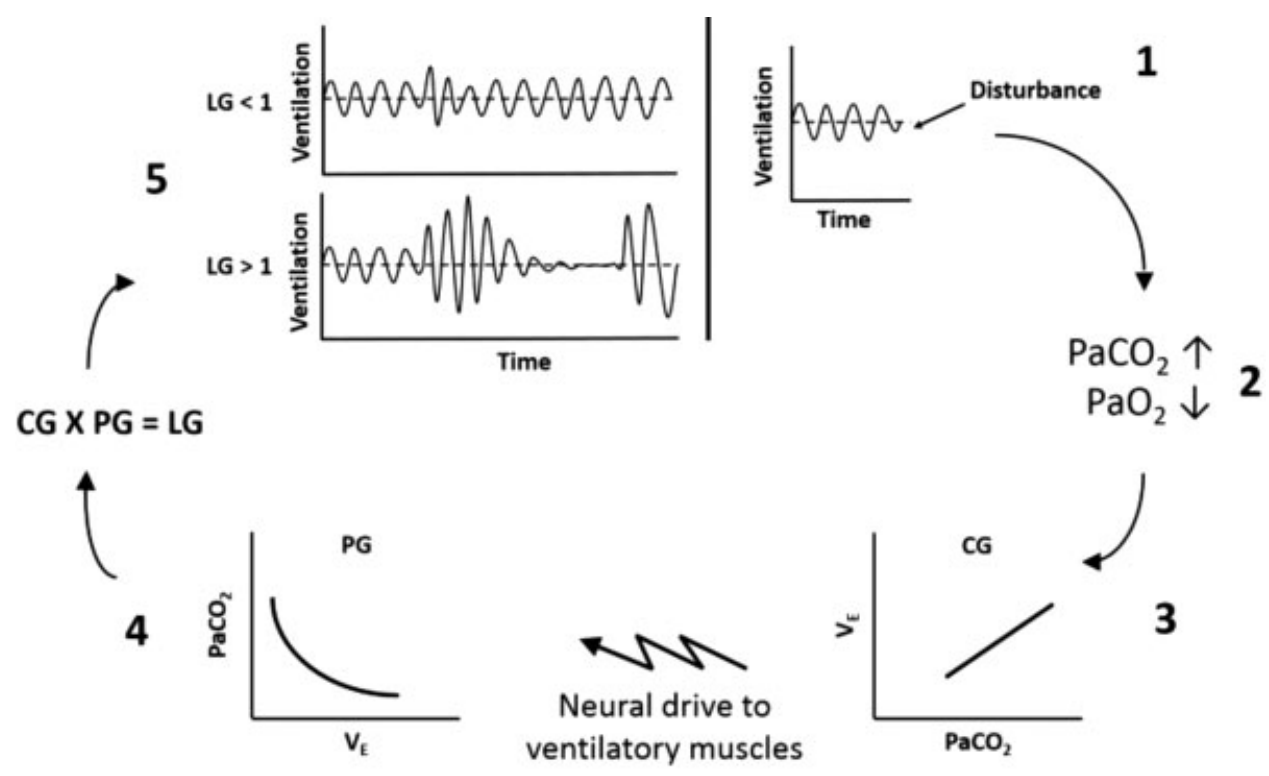

Fig. 2 Schematic of ventilatory loop gain. 1: a disturbance to breathing causes a reduction in ventilation below eupnea. 2: reduced ventilation increases arterial $\mathrm{CO}_{2}\left(\mathrm{PaCO}_{2}\right)$ and reduces arterial $\mathrm{O}_{2}\left(\mathrm{PaO}_{2}\right)$. 3: controller gain (CG) reflects the sensitivity of the peripheral and central chemoreceptors to blood gases and dictates the magnitude of neural drive to ventilatory muscles $\left(\Delta V_{\mathrm{E}} / \Delta \mathrm{PaCO} 2\right) .4$ : plant gain $(\mathrm{PG})$ represents the effectiveness of the lungs to change blood gases $\left(\Delta \mathrm{PaCO} / \Delta V_{\mathrm{E}}\right)$. 5: the product of $C G$ and PG determines overall loop gain (LG). If $L G$ is less than $1(\mathrm{LG}<1)$, the fluctuations in ventilation will dampen out and breathing will stabilize. If LG is greater than 1 (LG > 1 ), the fluctuations in ventilation will increase in amplitude and instability will be self-perpetuating. Reproduced with permission from Deacon-Diaz et al. 47 

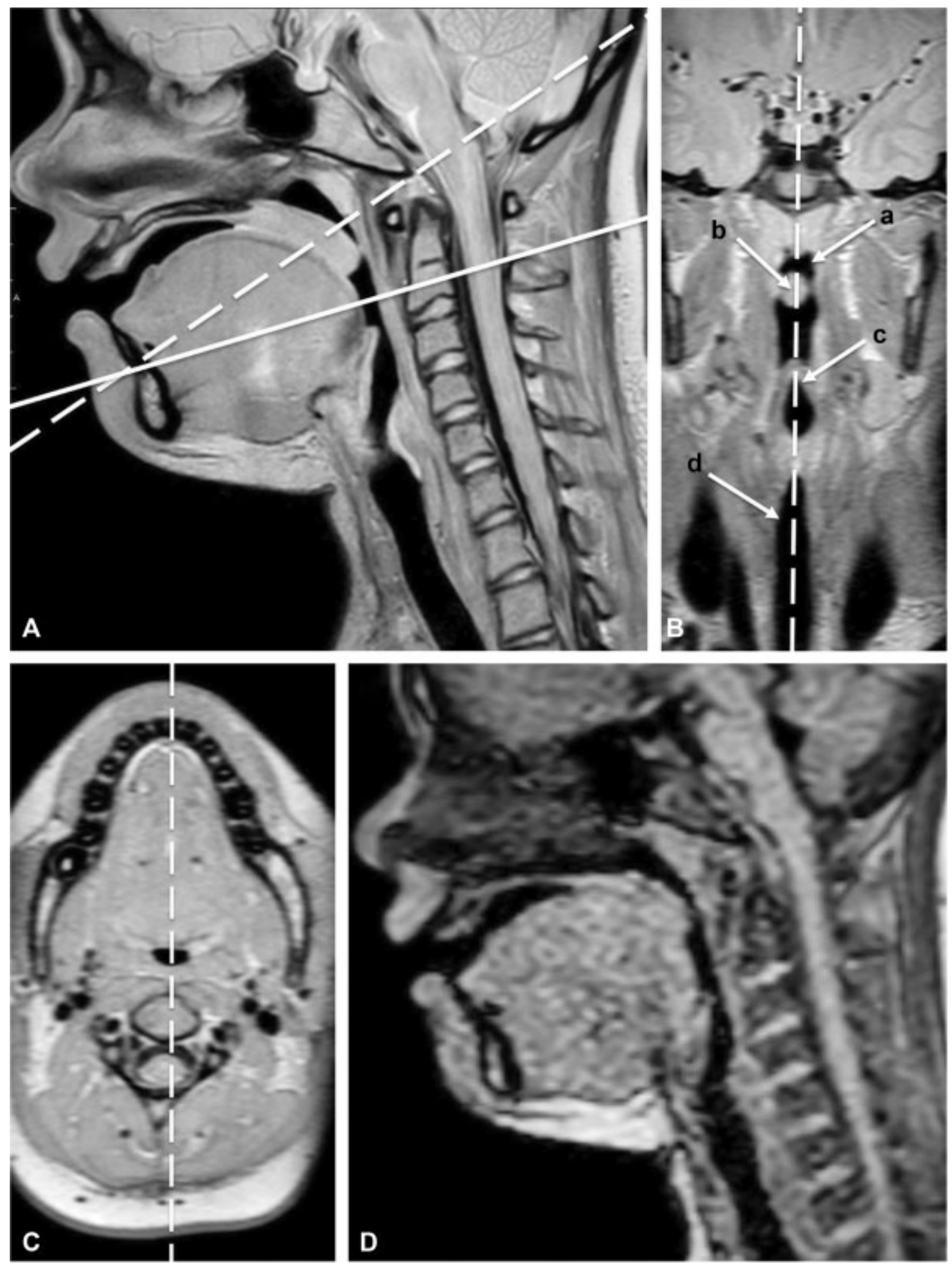

Fig. 3 MRI of a 15-year-old girl with Down's syndrome and persistent obstructive sleep apnea after lingual tonsillectomy, with an apneahypopnea index of 5.1 events per hour. (A): Midline sagittal three-dimensional proton-density respiratory-triggered image with 0.8 -mm isotropic resolution. Solid line depicts placement of imaging plane for cine of the retroglossal airway, and the dashed line shows placement of the plane for cine imaging of nasopharyngeal airway. (B) Coronal reformat of the data from (A) shows the length of the airway from the nasopharynx (a), uvula/soft palate (b), epiglottis (c), to subglottic trachea (d). Dashed line shows the orientation of the midline sagittal plane for cine of the upper airway. Using the reformatted image allows adjustment of the imaging plane so that the plane bisects the airway along the entire length, which can depict any dynamic multilevel obstruction that might occur. (C) Axial reformat of the data from the solid line in (A) shows the midline sagittal plane oriented to the midretroglossal airway and apex of the mandible. Children can rotate their heads during imaging; therefore, all planes should be checked on two images. (D) Sagittal midline cine gradient echo sequence available as a movie online shows soft tissue movement at the base of the tongue, causing intermittent obstruction at the level of the epiglottis. (Reproduced with permission of authors.)

for sedation, which does not cause significant respiratory depression. ${ }^{52}$

Currently, the expert opinion states that a 50\% collapse of the airway is abnormal and that $80 \%$ is very abnormal and should prompt further evaluation. Chen et al also showed that upper airway loop gain (UALG) could identify anatomi- cal risk factors for OSA. ${ }^{53}$ In this study, UALG was determined by measuring the ratio of the change in area drop to the change in area overshoot before and after airway collapse. Although patients with OSA did not have significantly different UALG $(n=4)$ from controls $(n=3)$, patients with higher AHI tended to have higher UALG values. Additionally, 
functional upper airway area did differ significantly between OSA patients and controls, suggesting simultaneous multislice real-time MRI (SMS RT-MRI) as a valuable tool to assess anatomy and physiology of patients with OSA. Therefore, UALG is both a measurement of airway collapse and a response to airway collapse that can be used to evaluate anatomy and pathophysiology of OSA patients. ${ }^{53}$

\section{Drug-Induced Sleep Endoscopy}

Finally, DISE has evolved to become a valuable tool for the evaluation of upper airway obstruction. Specifically, this noninvasive procedure allows clinicians to precisely locate points of obstruction during medication-induced sleep. Controversy exists regarding which anesthetic drugs are best mimickers of natural sleep, and hence there is no standardized protocol. When anesthetized, the patient's sleep stage can be approximated using the bispectral index score. Finally, the endoscope should be introduced through the nasal cavity, allowing visualization of the retrovelar, oropharyngeal, retrobasilingual, retroepiglottic, and glottic regions. ${ }^{54}$ If done correctly under optimal anesthesia to mimic natural sleep, DISE procedure can provide a comprehensive evaluation of the multisite obstruction in the upper airway. The site and the severity of the upper airway obstruction can be assessed based on the VOTE classification mentioned previously. ${ }^{55}$

\section{Conclusion}

In conclusion, airway development starting in utero through adolescence has a significant impact on the normal development of upper airway anatomy and physiology. Patients who present with minor signs of upper airway obstruction (i.e., snoring and adenoid facies) should be evaluated closely in the clinic, and studies assessing the degree of obstruction (polysomnography), upper airway anatomy (imaging studies), and function (DISE, UALG) should be performed to identify the site and severity of upper airway obstruction. These complementary and informative approaches will allow physicians to accurately phenotype upper airways, leading to a targeted and personalized treatment strategy and to treat, cure, or potentially prevent OSA before it can have its lasting effects on the child's overall health and quality of life.

\section{Conflict of Interest}

None declared.

\section{Reference}

1 American Thoracic Society. Standards and indications for cardiopulmonary sleep studies in children. Am J Respir Crit Care Med 1996;153(02):866-878

2 Maris M, Verhulst S, Wojciechowski M, Van de Heyning P, Boudewyns A. Prevalence of obstructive sleep apnea in children with Down syndrome. Sleep (Basel) 2016;39(03):699-704

3 Kaditis AG, Alonso Alvarez ML, Boudewyns A, et al. Obstructive sleep disordered breathing in 2- to 18-year-old children: diagnosis and management. Eur Respir J 2016;47(01):69-94

4 Ingram DG, Singh AV, Ehsan Z, Birnbaum BF. Obstructive sleep apnea and pulmonary hypertension in children. Paediatr Respir Rev 2017;23:33-39
5 Loughlin GM, Wynne JW, Victorica BE. Sleep apnea as a possible cause of pulmonary hypertension in Down syndrome. J Pediatr 1981;98(03):435-437

6 Blunden S, Lushington K, Kennedy D, Martin J, Dawson D. Behavior and neurocognitive performance in children aged 5-10 years who snore compared to controls. J Clin Exp Neuropsychol 2000;22 (05):554-568

7 Marcus CL, Brooks LJ, Draper KA, et al; American Academy of Pediatrics. Diagnosis and management of childhood obstructive sleep apnea syndrome. Pediatrics 2012;130(03): 576-584

8 Guilleminault C, Li K, Quo S, Inouye RN. A prospective study on the surgical outcomes of children with sleep-disordered breathing. Sleep 2004;27(01):95-100

9 Tauman R, Gulliver TE, Krishna J, et al. Persistence of obstructive sleep apnea syndrome in children after adenotonsillectomy.J Pediatr 2006;149(06):803-808

10 Bhattacharjee R, Kheirandish-Gozal L, Spruyt K, et al. Adenotonsillectomy outcomes in treatment of obstructive sleep apnea in children: a multicenter retrospective study. Am J Respir Crit Care Med 2010;182(05):676-683

11 Guilleminault C, Huang YS. From oral facial dysfunction to dysmorphism and the onset of pediatric OSA. Sleep Med Rev 2018;40:203-214

12 Montgomery-Downs HE, Young ME, Ross MA, Polak MJ, Ritchie SK, Lynch SK. Sleep-disordered breathing symptoms frequency and growth among prematurely born infants. Sleep Med 2010;11 (03):263-267

13 Rosen CL, Larkin EK, Kirchner HL, et al. Prevalence and risk factors for sleep-disordered breathing in 8- to 11-year-old children: association with race and prematurity. J Pediatr 2003;142(04): 383-389

14 Huang YS, Guilleminault C. Pediatric obstructive sleep apnea and the critical role of oral-facial growth: evidences. Front Neurol 2013; 3:184

15 Ling L, Olson EB Jr, Vidruk EH, Mitchell GS. Developmental plasticity of the hypoxic ventilatory response. Respir Physiol 1997; 110(2-3):261-268

16 Kheirandish-Gozal L, Gozal D. Sleep Disordered Breathing in Children: A Comprehensive Clinical Guide to Evaluation and Treatment. New York, NY: Humana Press; 2012

17 Pendolino AL, Lund VJ, Nardello E, Ottaviano G. The nasal cycle: a comprehensive review. Rhinol Online 2018;1:10

18 Mahdavinia M, Schleimer RP, Keshavarzian A. Sleep disruption in chronic rhinosinusitis. Expert Rev Anti Infect Ther 2017;15(05): 457-465

19 Kheirandish-Gozal L, Gozal D. Intranasal budesonide treatment for children with mild obstructive sleep apnea syndrome. Pediatrics 2008;122(01):e149-e155

20 Brouillette RT, Manoukian JJ, Ducharme FM, et al. Efficacy of fluticasone nasal spray for pediatric obstructive sleep apnea. J Pediatr 2001; 138(06):838-844

21 Peltomäki T. The effect of mode of breathing on craniofacial growth-revisited. Eur J Orthod 2007;29(05):426-429

22 Kezirian EJ, Hohenhorst W, de Vries N. Drug-induced sleep endoscopy: the VOTE classification. Eur Arch Otorhinolaryngol 2011;268(08):1233-1236

23 Azarbarzin A, Sands SA, Marques M, et al. Palatal prolapse as a signature of expiratory flow limitation and inspiratory palatal collapse in patients with obstructive sleep apnoea. Eur Respir J 2018;51(02):1701419

24 Genta PR, Sands SA, Butler JP, et al. Airflow shape is associated with the pharyngeal structure causing OSA. Chest 2017;152(03): 537-546

25 Isono S, Feroah TR, Hajduk EA, Brant R, Whitelaw WA, Remmers JE. Interaction of cross-sectional area, driving pressure, and airflow of passive velopharynx. J Appl Physiol (1985) 1997;83(03): 851-859 
26 Isono S, Remmers JE, Tanaka A, Sho Y, Sato J, Nishino T. Anatomy of pharynx in patients with obstructive sleep apnea and in normal subjects. J Appl Physiol (1985) 1997;82(04):1319-1326

27 Patil SP, Schneider H, Marx JJ, Gladmon E, Schwartz AR, Smith PL. Neuromechanical control of upper airway patency during sleep. J Appl Physiol (1985) 2007;102(02):547-556

28 Fogel RB, Malhotra A, Shea SA, Edwards JK, White DP. Reduced genioglossal activity with upper airway anesthesia in awake patients with OSA. J Appl Physiol (1985) 2000;88(04):1346-1354

29 Hotwani K, Sharma K, Jaiswal A. Evaluation of tongue/mandible volume ratio in children with obstructive sleep apnea. Dental Press J Orthod 2018;23(04):72-78

30 Lal C, White DR, Joseph JE, van Bakergem K, LaRosa A. Sleepdisordered breathing in Down syndrome. Chest 2015;147(02): 570-579

31 Donnelly LF, Shott SR, LaRose CR, Chini BA, Amin RS. Causes of persistent obstructive sleep apnea despite previous tonsillectomy and adenoidectomy in children with down syndrome as depicted on static and dynamic cine MRI. AJR Am J Roentgenol 2004;183 (01):175-181

32 Whittle AT, Marshall I, Mortimore IL, Wraith PK, Sellar RJ, Douglas NJ. Neck soft tissue and fat distribution: comparison between normal men and women by magnetic resonance imaging. Thorax 1999;54(04):323-328

33 Schwab RJ. Upper airway imaging. Clin Chest Med 1998;19(01): 33-54

34 Torre C, Camacho M, Liu SY, Huon LK, Capasso R. Epiglottis collapse in adult obstructive sleep apnea: a systematic review. Laryngoscope 2016;126(02):515-523

35 Luzzi V, Di Carlo G, Saccucci M, et al. Craniofacial morphology and airflow in children with primary snoring. Eur Rev Med Pharmacol Sci 2016;20(19):3965-3971

36 Pacheco MC, Fiorott BS, Finck NS, Araújo MT. Craniofacial changes and symptoms of sleep-disordered breathing in healthy children. Dental Press J Orthod 2015;20(03):80-87

37 Lee RW, Chan AS, Grunstein RR, Cistulli PA. Craniofacial phenotyping in obstructive sleep apnea-a novel quantitative photographic approach. Sleep 2009;32(01):37-45

38 Pirilä-Parkkinen K, Pirttiniemi P, Nieminen P, Tolonen U, Pelttari U, Löppönen H. Dental arch morphology in children with sleepdisordered breathing. Eur J Orthod 2009;31(02):160-167

39 Berthon-Jones M, Sullivan CE. Ventilation and arousal responses to hypercapnia in normal sleeping humans. J Appl Physiol 1984; 57(01):59-67

40 Osman AM, Carter SG, Carberry JC, Eckert DJ. Obstructive sleep apnea: current perspectives. Nat Sci Sleep 2018;10:21-34

41 Smith CA, Chenuel BJ, Henderson KS, Dempsey JA. The apneic threshold during non-REM sleep in dogs: sensitivity of carotid body vs. central chemoreceptors. J Appl Physiol (1985) 2007;103 (02):578-586

42 Bandla P, Huang J, Karamessinis L, et al. Puberty and upper airway dynamics during sleep. Sleep 2008;31(04):534-541

43 Huang J, Karamessinis LR, Pepe ME, et al. Upper airway collapsibility during REM sleep in children with the obstructive sleep apnea syndrome. Sleep 2009;32(09):1173-1181

44 Wilhelm CP, deShazo RD, Tamanna S, Ullah MI, Skipworth LB. The nose, upper airway, and obstructive sleep apnea. Ann Allergy Asthma Immunol 2015;115(02):96-102

45 Ahbab S, Ataoğlu HE, Tuna M, et al. Neck circumference, metabolic syndrome and obstructive sleep apnea syndrome; evaluation of possible linkage. Med Sci Monit 2013;19:111-117

46 Isono S, Tanaka A, Tagaito Y, Ishikawa T, Nishino T. Influences of head positions and bite opening on collapsibility of the passive pharynx. J Appl Physiol (1985) 2004;97(01):339-346

47 Deacon-Diaz N, Malhotra A. Inherent vs. induced loop gain abnormalities in obstructive sleep apnea. Front Neurol 2018;9:896

48 Loewen A, Ostrowski M, Laprairie J, et al. Determinants of ventilatory instability in obstructive sleep apnea: inherent or acquired? Sleep 2009;32(10):1355-1365

49 Salloum A, Rowley JA, Mateika JH, Chowdhuri S, Omran Q, Badr MS. Increased propensity for central apnea in patients with obstructive sleep apnea: effect of nasal continuous positive airway pressure. Am J Respir Crit Care Med 2010;181(02):189-193

50 Mateika JH, Mendello C, Obeid D, Badr MS. Peripheral chemoreflex responsiveness is increased at elevated levels of carbon dioxide after episodic hypoxia in awake humans. J Appl Physiol (1985) 2004;96(03):1197-1205, discussion 1196

51 Sands SA, Terrill PI, Edwards BA, et al. Quantifying the arousal threshold using polysomnography in obstructive sleep apnea. Sleep (Basel) 2018;41(01):

52 Patini R, Arrica M, Di Stasio E, Gallenzi P, Cordaro M. The use of magnetic resonance imaging in the evaluation of upper airway structures in paediatric obstructive sleep apnoea syndrome: a systematic review and meta-analysis. Dentomaxillofac Radiol 2016;45(07):20160136

53 Fleck RJ, Shott SR, Mahmoud M, Ishman SL, Amin RS, Donnelly LF. Magnetic resonance imaging of obstructive sleep apnea in children. Pediatr Radiol 2018;48(09):1223-1233

54 Chen W, Gillett E, Khoo MCK, Davidson Ward SL, Nayak KS. Realtime multislice MRI during continuous positive airway pressure reveals upper airway response to pressure change. J Magn Reson Imaging 2017;46(05):1400-1408

55 Blumen M, Bequignon E, Chabolle F. Drug-induced sleep endoscopy: a new gold standard for evaluating OSAS? Part I: technique. Eur Ann Otorhinolaryngol Head Neck Dis 2017;134(02): 101-107 